\title{
LINC00992 contributes to the oncogenic phenotypes in prostate cancer via targeting miR-3935 and augmenting GOLM1 expression
}

Jianheng Chen ${ }^{1}$, Xiaodong Liư ${ }^{1}$, Kunbin Ke ${ }^{1}$, Jianan Zou², Zhan Gao ${ }^{3}$, Tomonori Habuchi ${ }^{4}$ and Xuezhen Yang ${ }^{5^{*}}$ (1)

\begin{abstract}
Background: Accumulating evidence has revealed the critical role of long non-coding RNAs (IncRNAs) in cellular processes during tumor progression. As documented in cancer-related literatures, LINC00992 expression is associated with cancer progression, whereas its function in tumors including prostate cancer has not been characterized yet.

Methods: Data from GEPIA database suggested LINC00992 expression in prostate cancer tissues. The expression levels of RNAs were monitored via qRT-PCR. Western blot evaluated the levels of proteins. The proliferation, apoptosis and migration of prostate cancer cells were assessed by CCK-8, EdU, TUNEL, Transwell and wound healing assays. Luciferase reporter, RNA pull down and RIP assays were applied to detect the interplays among LINC00992, miR-3935 and GOLM1.

Results: Elevated levels of LINC00992 and GOLM1 were detected in prostate cancer tissues and cells. LINC00992 exerted facilitating functions in prostate cancer cell proliferation and migration. Mechanically, LINC00992 interacted with and negatively regulated miR-3935 to elevate GOLM1 expression in prostate cancer cells. In addition, the in vitro suppressive effect of silenced LINC00992 on prostate cancer cell proliferation and migration was reversed by GOLM1 upregulation. Likewise, LINC00992 depletion restrained tumor growth in vivo was offset by enhanced GOLM1 expression.

Conclusions: LINC00992 competitively bound with miR-3935 to elevate GOLM1 expression and therefore facilitate the oncogenic phenotypes of prostate cancer cells, implying a potential LINC00992-targeted therapy for prostate cancer.
\end{abstract}

Keywords: INC00992, miR-3935, GOLM1, Prostate cancer

\footnotetext{
* Correspondence: engineyang@sina.com

${ }^{5}$ Department of Urology, the Second Affiliated Hospital of Bengbu Medical College, 220 Hongye Road, Bengbu 233000, Anhui, China

Full list of author information is available at the end of the article
}

(c) The Author(s). 2020 Open Access This article is licensed under a Creative Commons Attribution 4.0 International License, which permits use, sharing, adaptation, distribution and reproduction in any medium or format, as long as you give appropriate credit to the original author(s) and the source, provide a link to the Creative Commons licence, and indicate if changes were made. The images or other third party material in this article are included in the article's Creative Commons licence, unless indicated otherwise in a credit line to the material. If material is not included in the article's Creative Commons licence and your intended use is not permitted by statutory regulation or exceeds the permitted use, you will need to obtain permission directly from the copyright holder. To view a copy of this licence, visit http://creativecommons.org/licenses/by/4.0/. The Creative Commons Public Domain Dedication waiver (http://creativecommons.org/publicdomain/zero/1.0/) applies to the data made available in this article, unless otherwise stated in a credit line to the data. 


\section{Background}

Clinically, prostate cancer manifests as a dominating cause of male-related death worldwide and is characterized as the most continually occurred tumor among men in the United States [1]. The biggest challenge is the detectable bone metastases in roughly $80 \%$ advanced prostate cancer [2]. Virtually all prostate cancer patients during 2 years' androgen deprivation treatment inevitably undergo castration-resistance, which contributes to the poor clinical consequences in prostate cancer [3]. However, the mechanism underlaid prostate cancer remains mostly unknown.

The widely studied long noncoding RNAs (lncRNAs) are transcribed from non-protein-coding human genome and have more than $200 \mathrm{nt}$ in length [4]. LncRNAs are increasingly functionally identified and experimentally consolidated to be related to tumor neoplasia and progression in diverse cancers [5]. Additionally, lncRNAs with dysregulation can functionally modulate tumor development from multiple pathological aspects such as cell proliferation, drug-resistance, and metastasis [6-8]. For example, lncRNA A1BG-AS1 inhibits cell proliferation and invasion in hepatocellular carcinoma via targeting miR-216a-5p [9]. LncRNA LOC730100 sponges miR-760 from FOXA1 to accelerate cell proliferation and invasion in glioma [10]. LncRNA SNHG16 functions as an oncogene in hepatocellular carcinoma [11]. Long intergenic non-protein coding RNA 992 (LINC00992) is a novel lncRNA that has been previously revealed to be elevated in tumors and substantiated as a master regulator for chemo-resistance [12]. Besides, LINC00992 has been uncovered as an elevated IncRNA in prostate cancer [13], which is consistent with the detection from GEPIA database. Despite that, no previous study has given a comprehensive explanation about the precise function or detailed mechanism of LINC00992 in prostate cancer.

In past decades, the fact that IncRNAs function in tumors depending on their secondary or tertiary structures has been reported in many cancer-linked studies. For instance, in the nucleus, lncRNAs are entitled to work as molecular scaffolds or alternative splicing assistants [14, 15]. On the contrary, lncRNAs dispersing in cytoplasm influence downstream mRNA translation or degradation through serving as miRNA sponges $[16,17]$. For example, TNF- $\alpha$-induced IncRNA LOC105374902 promotes the malignant behaviors of cervical cancer cells by acting as a sponge of miR-1285-3p [18]. LncRNA TTN-AS1 promotes papillary thyroid cancer tumorigenesis by regulating miR-153-3p/ZNRF2 axis [19]. Nevertheless, whether LINC00992 could exert its functions in prostate cancer via its sponging role of certain miRNA remains unknown.

We conducted this research aiming to explore the function or probable mechanism of LINC00992 in prostate tumor, which might enrich the understanding in terms of prostate tumor pathology and contribute to a wider applied scope.

\section{Methods \\ Tissue samples}

The prostate cancer tissue samples and matched peritumor tissue samples were collected from 60 patients diagnosed with prostate cancer, under the approval of the Ethics Committee of the First Affiliated Hospital of Kunming Medical University. Each participant did not receive radiotherapy and chemotherapy prior to tissue collection, and signed the written informed consents before this study. All samples were snap-frozen in liquid nitrogen and then stored at $-80^{\circ} \mathrm{C}$ until required for further analysis.

\section{Cell culture}

The prostate epithelial cell line (RWPE-1; CRL-11609) and prostate cancer cells, including PC3 (CRL-1435), LNCaP (CRL-1740), C4-2 (CRL-3314), and DU145 (HTB-81), were all purchased from American Type Culture Collection (ATCC; Manassas, VA, USA) in October 2017. All cells were cultured as recommended in Dulbecco's modified Eagle's medium containing 10\% FBS (GIBCO, MA, USA) under the condition of a cell incubator with $5 \% \mathrm{CO}_{2}$ at $37^{\circ} \mathrm{C}$. Before using in this study, all cell lines were authenticated by STR profiling and tested for mycoplasma contamination in June 2018.

\section{Cell transfection}

LINC00992 shRNA or negative control shRNA, and pcDNA3.1/LINC00992, pcDNA3.1/GOLM1 or its empty control pcDNA3.1 plasmid were chemically synthesized and provided by Gene Pharma (Shanghai, China). MiR3935 mimics, miR-3935 inhibitor and their related negative controls (NC-mimics, NC-inhibitor) were all purchased for upregulating or downregulating miR-3935 from Ribobio (Guangzhou, China). In line with the directions of LipofectamineTM RNAiMAX Transfection Reagent (Thermo Fisher Scientific), transfection of these plasmids into DU145, PC3 and RWPE-1 cells was conducted and qRT-PCR checked the transfection efficiency. The sequences were as follows: sh-NC: 5 '-CCGG TAGTAATTGACAACCATTATACTCGAGTATAATG GTTGTCAATTACTATTTTTG-3', sh-LINC00992\#1: 5' -CCGGATTATCCAAGAGTATTAACATCTCGAGA TGTTAATACTCTTGGATAATTTTTTG-3' , sh-LINC0 0992\#2: 5'-CCGGTGTTAGATGATCATTGAGGTGCT CGAGCACCTCAATGATCATCTAACATTTTTG-3', s h-LINC00992\#3: 5'-CCGGTTACCTAATCAGTAGAT GCAGCTCGAGCTGCATCTACTGATTAGGTAATTT TTG-3'; NC-mimics: 5'-UCAGGUAGGGCUCAAACC AACC-3' ', miR-3935 mimics: 5' -UGUAGAUACGAGCA CCAGCCAC-3'; NC-inhibitor: 5' 
GGUGCCACUUAG-3', miR-3935 inhibitor: 5'-GUGGC UGGUGCUCGUAUCUACA-3'.

\section{Quantitative real-time PCR (qRT-PCR)}

On the basis of the instructions of Trizol reagent (Invitrogen, USA), RNA extraction was executed in prostate cancer cells. After the examination of RNA purity with spectrophotometry, cDNA was obtained from above RNA with reverse transcription kit $(4,368,814$, Thermo Fisher Scientific, shanghai, China). qRT-PCR analysis was devised with the aid of a BioRad CFX96 system and SYBR green was applied for investigating the RNA levels. The internal reference for LINC00992 and mRNAs was GAPDH whereas that for miRNAs expression was U6. Relative expression was assessed based on the method of $2^{-\Delta \Delta C t}$.

\section{Western blot}

Protein content in cells was determined by western blot analysis. RIPA lysis buffer (Beyotime, Shanghai, China) was adopted for cell lysing, followed by the evaluation of the protein concentration with BCA Protein Assay Kit (P0011, Beyotime Tech.). 10\% SDS-PAGE gel was applied for separating proteins $(20 \mu \mathrm{g}$ protein per sample) and then proteins were transferred onto $0.2 \mu \mathrm{m}$ PVDF membranes (Bio-Rad, Hercules, CA, USA). Antibodies including anti-GOLM1 (ab109628, 1/1000; Abcam, Cambridge, UK), anti-PCNA (ab92552, 1/1000; Abcam), anti-CDK2 (ab32147, 1/1000; Abcam), anti-Cyclin D1 (ab40754, 1/1000; Abcam), anti-Bax (ab32503, 1/1000; Abcam), anti-Bcl-2 (ab32124, 1/1000; Abcam), antiMMP2 (ab97779, 1/1000; Abcam), anti-MMP9 (ab38898, 1/1000; Abcam), anti-p-Src (ab40660, 1/1000; Abcam), anti-Src (ab47405, 1/1000; Abcam), anti-p-FAK (ab81298, 1/1000; Abcam), anti-FAK (ab131435, 1/1000; Abcam), anti-GAPDH (ab8245, 1/1000; Abcam) and anti-Tubulin (ab7291, 1/10000, Abcam), were applied to probe the membranes overnight at $4{ }^{\circ} \mathrm{C}$. After that, the membranes were further incubated for $1 \mathrm{~h}$ with HRPconjugated secondary antibody (Santa Cruz, Co. Ltd., Sant Cruz, CA, USA) at room temperature. ECL Substrates (Millipore, Billerica, MA, USA) was utilized for the visualization of signals, followed by exposure to X-film (Kodak, Rochester, NY, USA). The quantification of immunoblots was conducted with the aid of imageJ software (National Institute of Health, Bethesda, MD, USA), with GAPDH or Tubulin as the normalizer as needed.

\section{Luciferase reporter assay}

Fragments of full-length LINC00992 with wild-type or mutant binding sites for miR-3935 and sequences of GOLM1 3'UTR containing wild-type or mutated miR3935 binding sites were inserted into the pmirGLO vectors (Promega, Madison, WI, USA) for the construction of reporters (LINC00992-WT, LINC00992-MUT, GOLM1-WT, GOLM1-MUT). Then, the four reporters and miR-3935 mimics or miR-3935 inhibitor (GenePharma) were co-transfected into DU145 and PC3 cells applying lipofectamine2000 (Invitrogen), as needed. Forty-eight hours later, Dual-Luciferase Reporter Assay System (Promega) was employed for the examination of the luciferase activity. GloMax ${ }^{\oplus}$ Discover Multimode Microplate Reader (Promega) assessed the ratio of Firefly/ Renilla luciferase activity and the activity of Renilla was the normalized control.

\section{RNA immunoprecipitation (RIP) assay}

According to the direction for usage of Magna $\mathrm{RIP}^{\mathrm{rm}}$ RNA Binding Protein Immunoprecipitation Kit (17-700, Millipore), RIP assay was strictly performed. RIP lysis buffer was firstly applied to treat the transfected DU145 and PC3 cells. Afterwards, the obtained cell lysates were processed with magnetic beads integrated with human anti-Ago2 antibodies (ab32381; Abcam, MA, USA) or anti-IgG (AP162-KC, Millipore). Following the recovery of antibody by the protein A/G beads, qRT-PCR detected the levels of LINC00992, miR-3935, and GOLM1 mRNA in the precipitates. IgG worked as the negative control for the normalization of RNA-IPs.

\section{RNA isolation of nuclear and cytoplasmic fractions}

The dispersion of LINC00992 in the prostate cancer cells was assayed as described previously [20]. The isolation of cytosolic and nuclear sections was executed following the protocol of PARIS ${ }^{\text {тм }}$ Kit (AM1921, Invitrogen). RNA levels of U1 (nuclear control), GAPDH (cytoplasmic control) and LINC00992, were all estimated by qRT-PCR analysis.

\section{Fluorescence in situ hybridization (FISH) assay}

In line with the recommendation of $\mathrm{Ribo}^{\text {тм }}$ FISH Kit (C10910, Ribobio, Guangzhou, China), FISH analysis was implemented for testing the presence of LINC00992 in prostate tumor cells. Ribobio Company synthesized the LINC00992 probes, labeled by Cy3 fluorescent dye. Following the fixation by $4 \%$ paraformaldehyde and $0.5 \%$ Triton X-100 permeabilization, DU145 and PC3 cells were subsequently blocked in pre-hybridization buffer/ blocking solution. Then incubation of cells with probe/ hybridization buffer was later performed. Next day, after rinsing and Hoechst staining, the fluorescence was measured under a confocal laser scanning microscope (Zeiss, Germany).

\section{Cell counting kit-8 (CCK-8) assay}

For the viability assessment in DU145, PC3 and RWPE1 cells, CCK- 8 assay was implemented as described 
previously [21]. Cell viability was monitored at $24,48,72$ and $96 \mathrm{~h}$. In short, after being seeded onto 96-well plates and cultured for indicated times, cells were processed with $10 \mu \mathrm{l}$ of CCK- 8 solution. Then, a microplate reader examined the absorbance values at the wavelength of $450 \mathrm{~nm}$.

\section{5'-ethynyl-2'-deoxyuridine (EdU) incorporation assay}

Cell proliferation was examined through EdU assay as described previously [22], by using Click-iT EdU Alexa Fluor 488 Imaging Kit (C10086, Invitrogen). After 48 h of transfection, EdU staining was carried out as instructed. The observation and calculation of EdUpositive cells was proceeded under the fluorescence microscopy.

\section{Transferase-mediated dUTP nick end labeling (TUNEL) staining}

TUNEL assay was carried out as described previously [23], for probing DU145 and PC3 cell apoptosis with the assistance of an In Situ Cell Death Detection Kit (11,684, 795,910, Roche, Mannheim, Germany). TUNEL-positive cells were recorded under a light microscope $(\times 400)$ from 10 visual fields which were chosen at random.

\section{Transwell migration assay}

The application of transwell chambers with pore size 8$\mu \mathrm{m}$ (Corning Costar, Cambridge, MA, USA) was aimed for detecting cell migration in strict line with the instructions. Cells that were previously suspended in serum-free RPMI-1640 media were seeded into the upper chamber. RPMI-1640 medium containing 20\% FBS was supplemented in lower chamber as a chemoattractant. Cells in the filters following $48 \mathrm{~h}$ incubation were immobilized in methanol and went through $0.1 \%$ crystal violet staining. The images of cells migrated through the filters were obtained and counted under the microscope.

\section{Wound healing assay}

The DU145, PC3 and RWPE- 1 cells $\left(2 \times 10^{3}\right.$ cells/well $)$ were prepared on glass culture dishes and cultivated at $37^{\circ} \mathrm{C}$ for a whole night to allow cells adhered to the plates, followed by the straight scratch made with a plastic pipette tip after cell samples reached $80 \%$ confluence. Later, cells were rinsed in PBS to clear the detached cells. Finally, the wounds at 0 and $24 \mathrm{~h}$ were imaged via a light microscopy (Olympus, Tokyo, Japan).

\section{In vivo experiment}

Sixteen six-week-old male BALB/C athymic nude mice were commercially available from the National Laboratory Animal Center (Beijing, China) and maintained in SPF-grade animal lab. All animal-related protocols were approved by the Animal Research Ethics Committee of the First Affiliated Hospital of Kunming Medical University. The in vivo experiment was undertaken via subcutaneous injection of $5 \times 10^{6}$ DU145 cells into the nude mice, while the DU145 cells injected into indicated four groups of mice were transfected with sh-NC, shLINC00992\#1, sh-LINC00992\#1 + pcDNA3.1, or shLINC00992\#1 + pcDNA3.1/GOLM1. Tumor volume was monitored every 4 days. 28-day after injection, nude mice were sacrificed via cervical dislocation and then tumor samples were carefully dissected for weight assessment and hematoxylin and eosin (H\&E) staining.

\section{Immunohistochemistry (IHC)}

The tumor samples collected from in vivo experiments were treated with $4 \%$ PFA, dehydrated and embedded in paraffin. Afterwards, the paraffin-embedded sections $(4 \mu \mathrm{m})$ were prepared for IHC assay as described previously [24], by use of the anti-Ki67 and anti-PCNA antibodies (Abcam).

\section{Statistical analysis}

SPSS 17.0 statistical software (SPSS, Armonk, NY, USA) was employed in the processing of data from three biological replicates, and data were expressed as mean \pm SD. Significance of difference within two groups was determined using Student's t-test, while that among no less than two groups was tested via one-way or two-way ANOVA. $P<0.05$ was considered as the threshold of significance.

\section{Results}

LINC00992 is overexpressed in prostate cancer and regulates cell proliferation, apoptosis and migration

LINC00992 expression pattern in prostate cancer was acquired from online GEPIA database. As a result, LINC00992 was considerably upregulated in PRAD (prostate adenocarcinoma) tissues relative to normal ones (Fig. 1a). After detecting LINC00992 expression in tissue samples obtained from patients with prostate cancer, we observed that LINC00992 expression was higher in prostate cancer tissues than that in peri-tumor tissues (Figure S1A). Moreover, clinical data showed that higher expression of LINC00992 in prostate cancer patients was associated with lower survival rate (Figure S1B). Furthermore, LINC00992 expression in the prostate cancer cells and RWPE-1 cells was evaluated by qRT-PCR. Consequently, higher level of LINC00992 was exhibited in prostate cancer cells than that in RWPE-1 cells (Fig. $1 b)$, which was completely consistent with the result presented in previous discovery [13]. Particularly, DU145 and PC3 cells expressed the highest level of LINC00992 and was thereby chosen for the later assays. For silencing LINC00992, special shRNAs targeting LINC00992 was 


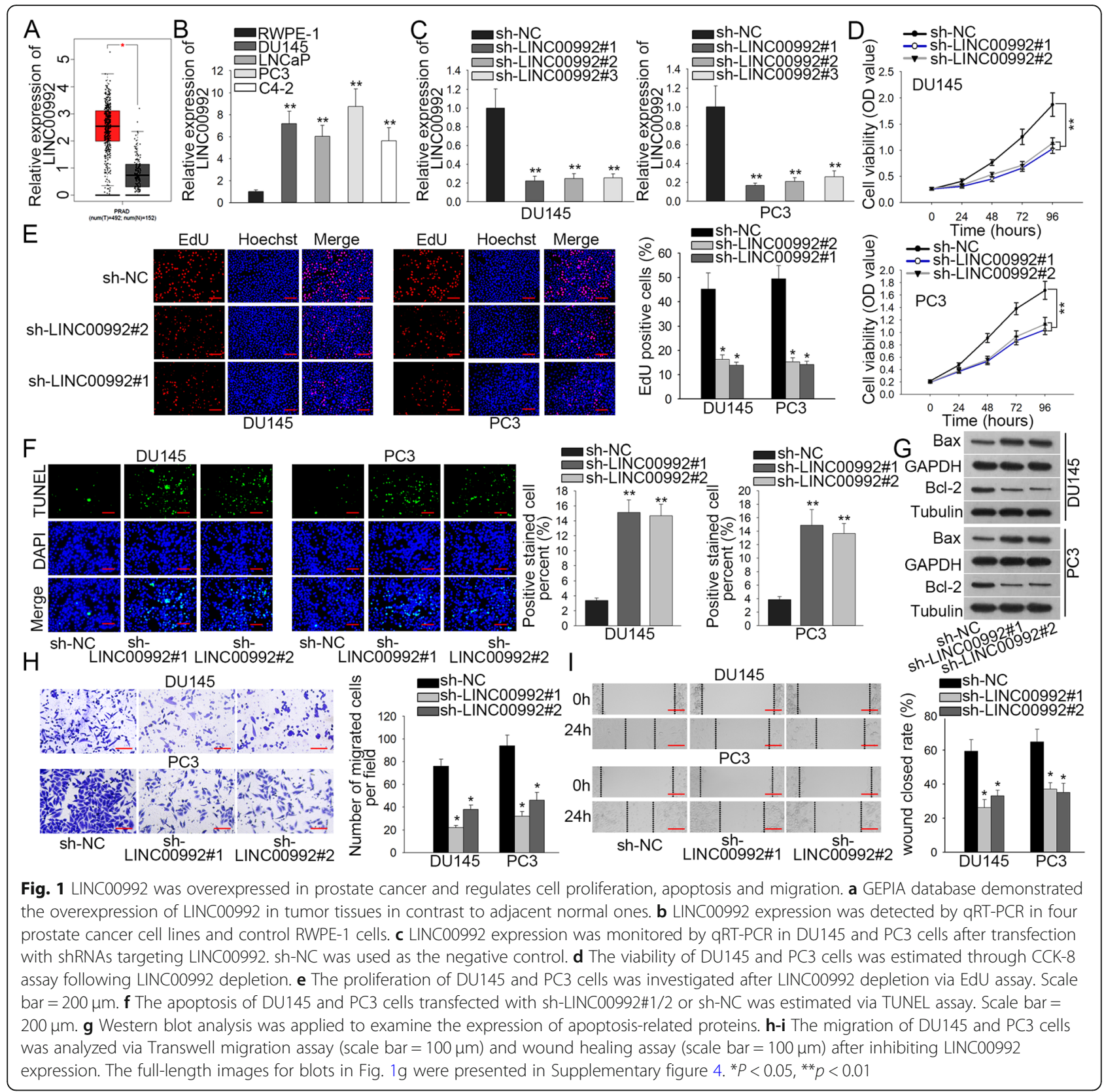

transfected into DU145 and PC3 cells and the efficiency was corroborated in qRT-PCR (Fig. 1c). And then the data from CCK-8 assay revealed that LINC00992 depletion suppressed the proliferation of DU145 and PC3 cells (Fig. 1d). As expected, a declined proportion of EdU positive cells was observed after knocking down LINC00992 (Fig. 1e), suggesting the suppressive effect of LINC00992 deficiency on prostate cancer cell proliferation. Additionally, the expression levels of proliferationrelated proteins (PCNA, CDK2 and Cyclin D1) were all reduced by silenced LINC00992 (Figure S1C). On the contrary, TUNEL assay uncovered that LINC00992 knockdown facilitated cell apoptosis (Fig. 1f). Meanwhile, western blot analysis revealed that LINC00992 knockdown promoted the apoptosis of DU145 and PC3 cells, as Bax protein level was increased whereas Bcl-2 protein level was decreased after LINC00992 was silenced in these two cells (Figs. 1g, Figure S1D). Further, Transwell and wound healing assays indicated that the migration of DU145 and PC3 cells was retarded by LINC00992 depletion (Fig. 1h-i). Likewise, the expression of migration-related molecular markers (MMP2, MMP9, p-Src and p-FAK) was decreased by LINC00992 inhibition (Figure S1E). To further verify the biological 
role of LINC00992 in prostate cancer, we carried out gain-of-function assays in RWPE-1 cells. After overexpressing LINC00992 in RWPE-1 cells (Figure S2A), cell proliferation was promoted (Figure S2B-C). As expected, the expression of PCNA, CDK2 and Cyclin D1 was decreased by upregulation of LINC00992 (Figure S2D). Similarly, LINC00992 upregulation facilitated cell migration (Figure S2E-F). In addition, upregulating LINC00992 resulted in the elevated protein levels of MMP2, MMP9, p-Src and p-FAK (Figure S2G). All these data elucidated that LINC00992 could facilitate cell proliferation and migration whereas suppress cell apoptosis in prostate cancer.

\section{MiR-3935 is targeted by LINC00992}

Given the high correlation of the sub-localization of LINC00992 with its functional mechanism, the predication of LINC00992 presence in cells was performed via LncLocator (http://www.csbio.sjtu.edu.cn/bioinf/lncLocator/). Result predicted that LINC00992 located mainly in cytoplasm (Fig. 2a). Likewise, FISH assay and RNA isolation of nuclear and cytoplasmic fractions further verified the abundance of LINC00992 in the cytoplasm of prostate cancer cells (Fig. 2b-c), highlighting a posttranscriptional control of LINC00992 in such cells. Hence, we speculated that LINC00992 might act as a ceRNA in prostate cancer regulation. According to DIANA-lncBase, the top three potential miRNAs possessing the binding capacity with LINC00992 were listed (Fig. 2d). To target the highly-matched miRNA to LINC00992, qRT-PCR analysis was conducted to test the expression changes of these miRNAs following either LINC00992 depletion or augmentation. The results demonstrated that only miR-3935 expression was increased by LINC00992 depletion (Fig. 2e), but reduced by LINC00992 overexpression in the meantime (Fig. 2f). Thus, miR-3935 was chosen for further analysis. Afterwards, RNA pull down assay was implemented and the result depicted that LINC00992 was pulled down by Bio-miR-3935-WT (Fig. 2g), which indicated the binding of LINC00992 and miR-3935. Later, we observed the satisfactory efficiency of miR-3935 overexpression and miR-3935 inhibition through qRT-PCR analysis (Fig. 2h). Thereafter, RIP assay applying anti-Ago2 was executed. Results illustrated that LINC00992 and miR-3935 were highly enriched in anti-Ago2 group in comparison with control antibody (Fig. 2i), certifying the association of LINC00992 with miR-3935 in the RNA-induced silencing complexes (RISCs). To further explore the interaction between LINC00992 and miR-3935, the binding sites between LINC00992 and miR-3935 were predicted at first, and then data from luciferase reporter assay revealed that miR-3935 upregulation decreased the luciferase activity of LINC00992-WT reporter whereas
miR-3935 inhibition increased the luciferase activity of LINC00992-WT reporter (Fig. 2j). Altogether, LINC0 0992 combined with miR-3935 to act as a miRNA decoy in prostate cancer.

\section{LINC00992 regulates the expression of GOLM1, a target of miR-3935}

Present evidence has suggested that miRNAs can bind with downstream target genes to inhibit their expression. Herein, we searched the miR-3935 target genes, and eight mRNAs were found out. Subsequently, we detected their expression in prostate cancer cells and normal cells. Interestingly, we found that only Golgi membrane protein 1 (GOLM1) was highly expressed in four prostate cancer cell lines relative to normal controls (Fig. 3a). Further, we discovered that GOLM1 expression was markedly upregulated in prostate cancer tissues according to data from GEPIA database (Fig. 3b). Similarly, GOLM1 expression was much higher in prostate cancer tissue samples than in peri-tumor samples (Figure S3A). In addition, the mRNA and protein levels of GOLM1 were overexpressed in prostate cancer cells in contrast to RWPE-1 cells (Fig. 3c, Figure S3B). Besides, GOLM1 has been previously revealed as a prostate cancer facilitator and was metastasis-related in prostate tumor [2529]. Thus, we hypothesized that GOLM1 might act as the downstream of LINC00992/miR-3935 signaling in prostate cancer. Through TargetScan (http://www.targetscan.org/vert_72/), the binding site between GOLM1 and miR-3935 was predicted (Fig. 3d). After conducting luciferase reporter assay in DU145 and PC3 cells, we observed that upregulation of miR-3935 specifically decreased the luciferase activity of GOLM1-WT reporter (Fig. 3e), confirming the interaction between miR-3935 and GOLM1 relied on the putative binding sites. Then, we unveiled that GOLM1 mRNA and protein levels were both reduced by LINC00992 inhibition or miR-3935 upregulation according to qRT-PCR and western blot analyses (Fig. 3f-g, Figure S3C). Moreover, data from RIP assay unveiled the binding of miR-3935 to GOLM1 in the RISCs (Fig. 3h). Further, we demonstrated that the decreased mRNA and protein levels of GOLM1 induced by LINC00992 depletion could be restored after inhibiting miR-3935 expression (Fig. 3i-j, Figure S3D). All the results showed that LINC00992 up-regulated GOLM1 expression via directly binding to miR-3935.

\section{LINC00992 promotes prostate cancer cell proliferation and migration via elevating GOLM1 expression}

To test whether LINC00992 affected prostate cancer cell proliferation, apoptosis and migration via regulating miR-3935-targeted GOLM1, we executed the rescue experiments with the up-regulation of GOLM1. To begin with, the efficiency of overexpressing GOLM1 was 


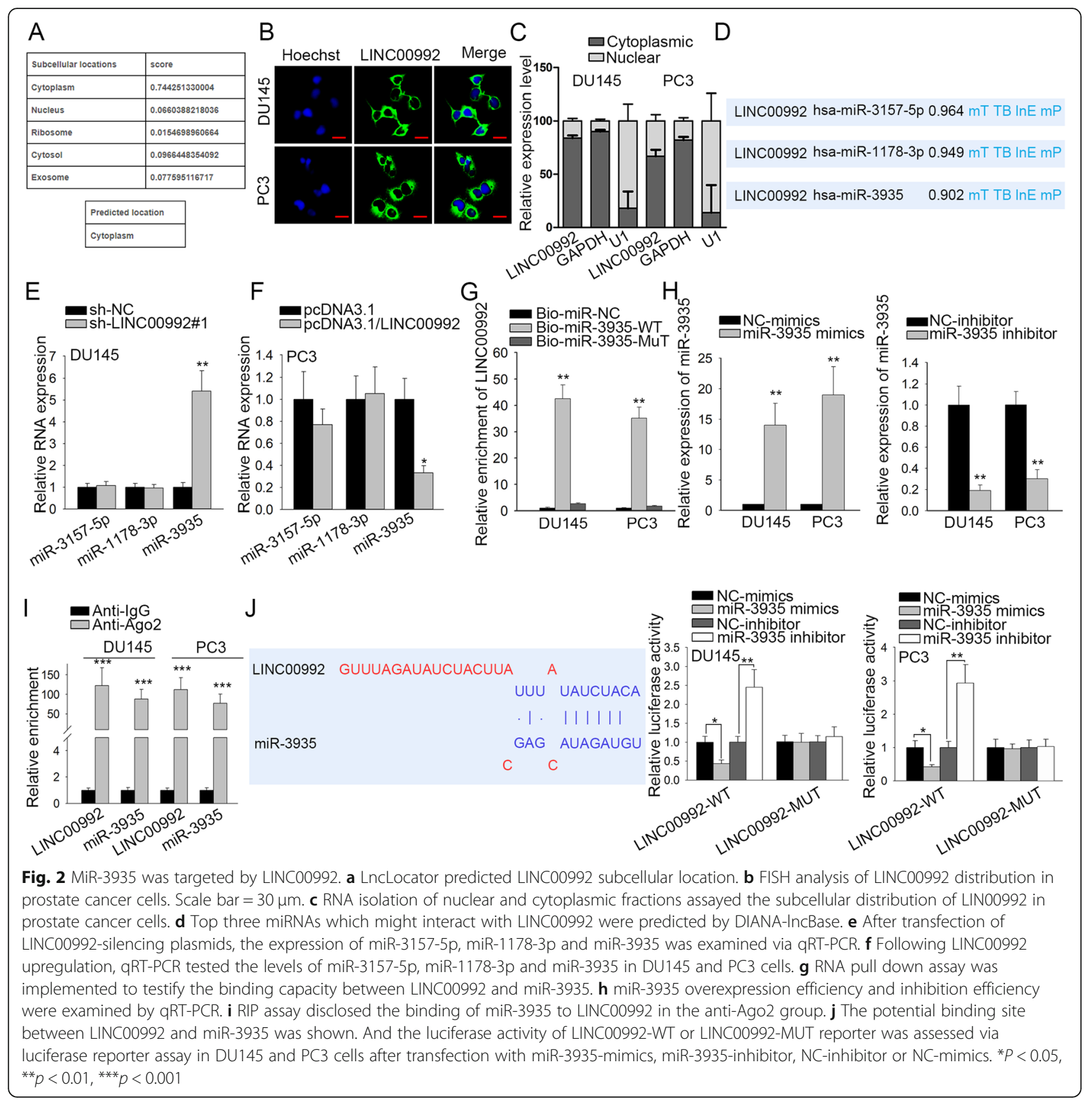

analyzed through qRT-PCR and western blot analyses and the outcome turned out to be satisfactory (Fig. 4a-b, Figure S3E). Then we observed that overexpression of GOLM1 could significantly elevate the mRNA and protein expression of GOLM1 in sh-LINC00992\#1-transfected cells (Figure S3F). Afterwards, data from CCK-8 revealed that the viability of DU145 cells was firstly hindered due to LINC00992 depletion while subsequent GOLM1 elevation reversed the inhibitory trend on DU145 cell viability (Fig. 4c). Results from EdU assay also exposed similar trends that GOLM1 up-regulation countervailed the suppressive impact posed by LINC00992 down-regulation on DU145 cell proliferation (Fig. 4d). Similarly, the restraining effect of silenced LINC00992 on the expression of proliferation-related proteins could be reversed by GOLM1 upregulation (Figure S3G). Later, TUNEL assay revealed that cell apoptosis rate was elevated by LINC00992 depletion and then overexpressing GOLM1 reduced the increased apoptosis rate of LINC00992-depleted cells (Fig. 4e). Likewise, western blot analysis uncovered that overexpressing GOLM1 could offset the effect of LINC00992 


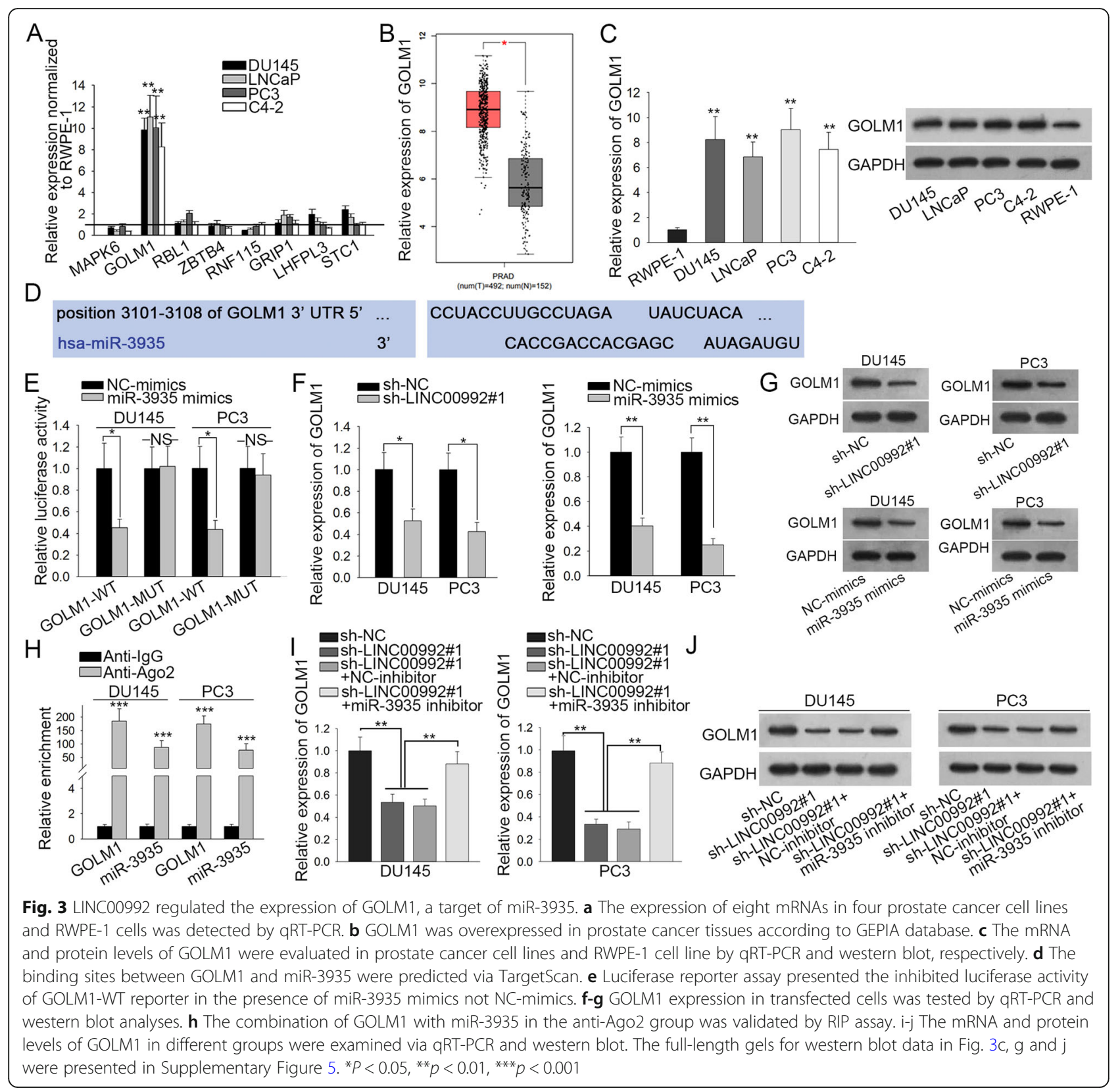

downregulation on the expression of apoptosis-related proteins (Fig. 4f, Figure S3H). Moreover, Transwell migration and wound healing assays illuminated that the retarding influence of silenced LINC00992 on cell migration could be rescued by GOLM1 overexpression (Fig. 4g-h). As expected, the inhibitory effect of LINC00992 depletion on the expression of migrationrelated molecular markers (MMP2, MMP9, p-Src and p-FAK) could be countervailed by GOLM1 overexpression (Figure S3I). Collectively, GOLM1 was required in LINC00992-regulated prostate cancer cellular processes.
LINC00992 contributes to tumor growth via upregulating GOLM1 expression

After the in vitro exploration of LINC00992 performance in prostate cancer, we applied the in vivo assays to further validate above findings. As shown in Fig. 5a, tumors derived from LINC00992-silenced DU145 cells were smaller, with the growth rate quite slower, than those from control cells; more importantly, such blockage on tumor growth was obviously countervailed after GOLM1 overexpression. Besides, elevating GOLM1 expression could recover the lessened tumor volume and declined tumor weight induced by LINC00992 deficiency 


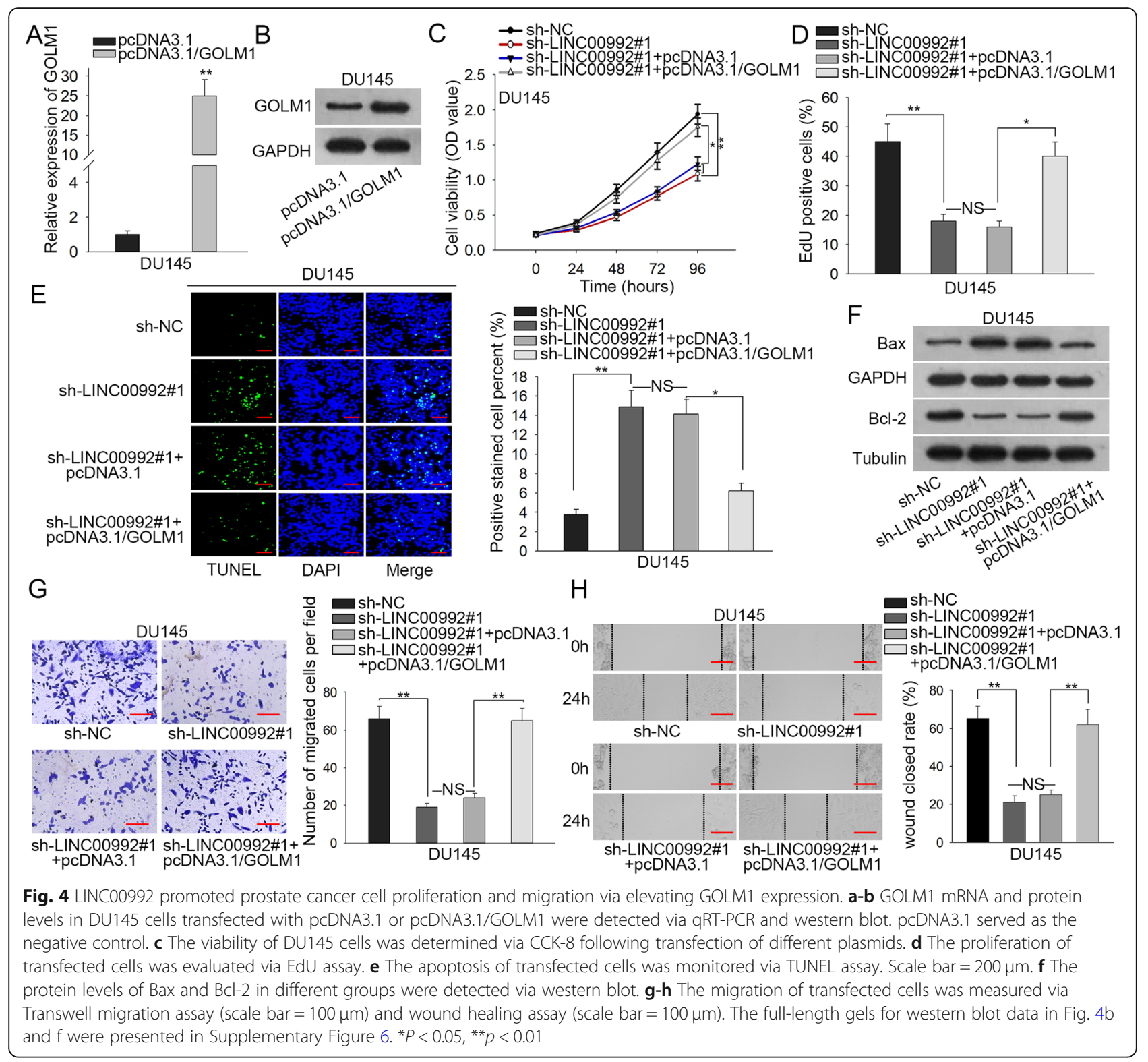

(Fig. 5b-c). Of note, we discovered decreased level of LINC00992 and enhanced level of miR-3935 in tumors from latter three groups compared to control group, while the lowered expression of GOLM1 in tumors with LINC00992 inhibition was normalized under GOLM1 overexpression (Fig. 5d). In addition, the inhibitory impact of silenced LINC00992 on the positivity of proliferationassociated proteins (PCNA and Ki67) could be reversed by upregulation of GOLM1 (Fig. 5e). Taken together, LINC00992 promoted the tumorigenesis of prostate cancer through upregulating GOLM1 expression.

\section{Discussion}

As documented, the aberrant regulation of lncRNAs is a frequent event in diversified tumor types. Besides, the correlation between abnormal lncRNA expression and prostate cancer oncogenesis has also been extensively explored. For example, lncRNA SNHG7 facilitates prostate cancer carcinogenesis via cyclin $\mathrm{D} 1$ by sponging miR-503 [30]. LncRNA SChLAP1 aggravates prostate cancer cell proliferation and metastasis by targeting miR-198 [31]. LncRNA PCAT-1 contributes to prostate cancer tumorigenesis through modulating FSCN1 and sponging miR-145-5p [32]. In our work, LINC00992 was revealed to be highly expressed in prostate cancer tissues and cells, but unlike former investigations, our study gave a precise explanation about its role in prostate cancer. Our study unveiled that LINC00992 promoted cell proliferation and migration, whereas suppressed cell apoptosis in prostate cancer. Above-mentioned data 


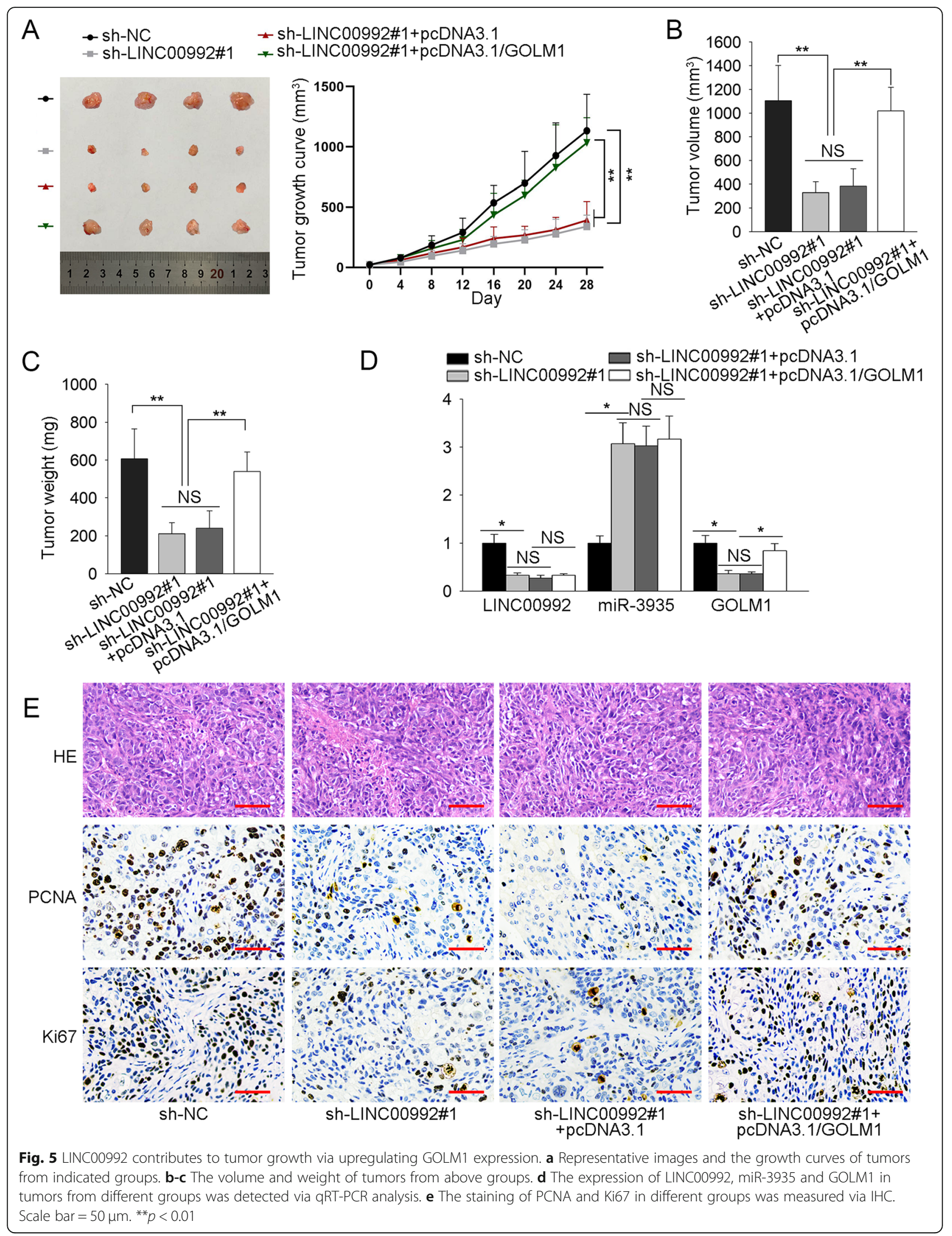


validated that LINC00992 elicited a tumor-promoting function in prostate cancer.

Presently, accumulating evidence has indicated that cytoplasmic lncRNAs assisted the expression of downstream miRNA-targeted mRNAs via sponging the specific miRNAs. Before exploring LINC00992-mediated mechanism in prostate cancer, herein we firstly discovered its subcellular distribution in prostate cancer cells with both aids from online prediction tool (LncLocator) and experimental data (FISH and RNA isolation of nuclear and cytoplasmic fractions). Our study for the first time uncovered that LINC00992 located mainly in the cytoplasm of prostate cancer cells. Besides, our study also completed LINC00992-modulated mechanism by disclosing the downstream target miR-3935. The direct interaction between LINC00992 and miR-3935 was also first validated in this research.

In the subsequent search of mRNA targeted by miR-3935, we paid heed to Golgi Membrane Protein 1 (GOLM1). Previously, downregulation of GOLM1 inhibits glioblastoma cell proliferation and motility, and progression [33, 34]. GOLM1 aggravates cell growth and metastasis in breast cancer, and aggressiveness in non-small-cell carcinoma and hepatocellular carcinoma [35-37]. In lung adenocarcinoma, GOLM1 facilitates cell proliferation and represents unfavorable survival $[38,39]$. In prostate cancer, GOLM1 has also been corroborated as tumor promoter [25-29]. Currently, we also revealed GOLM1 as upregulated in prostate cancer. Despite previously reported miRNAs targeting GOLM1 such as miR-382, miR-145, miR-200a, we first identified miR-3935 as the upstream regulator of GOLM1 in prostate tumor. Most importantly, rescue experiments highlighted that GOLM1 overexpression could rescue the suppressive effect of silenced LINC00992 on cell growth and migration in vitro and tumor growth in vivo.

\section{Conclusion}

To sum up, all findings presented in the work contributed to a better grasp about the mechanism in prostate cancer carcinogenesis. It was elaborated that LINC00992 accelerated prostate cancer cell growth and migration via sponging miR-3935 and therefore promoting GOLM1 expression, providing new promising therapeutic biomarkers for prostate cancer. Nevertheless, current study lacks the evidence confirming the regulation of LINC00992 on tumor metastasis in vivo, which will motivate us to explore further in the future study.

\section{Supplementary information}

Supplementary information accompanies this paper at https://doi.org/10. 1186/s12885-020-07141-4.
Additional file 1: Supplementary Figure 1. (A) GRT-PCR analysis indicated the upregulation of LINC00992 in prostate cancer tissue samples in contrast to peri-tumor samples. (B) The association of LINC00992 expression with overall survival of prostate cancer patients was analyzed via Kaplan-Meier curve. (C) The expression of proliferation-related proteins in transfected cells was evaluated via western blot. (D) The quantification of immunoblots in Fig. $1 \mathrm{~g}$ was shown. (E) The expression of migrationrelated molecular markers in transfected cells was analyzed via western blot. The full-length gels for western blot data in Figures S1C and S1E were presented in Supplementary Figure $7 .{ }^{* *} p<0.01$.

Additional file 2: Supplementary Figure 2. (A) The efficiency of LINC00992 overexpression was assessed via qRT-PCR in RWPE-1 cells. (BC) The proliferation ability of transfected cells was estimated via CCK-8 and EdU assays (scale bar $=200 \mu \mathrm{m}$ ). (D) The expression of proliferationrelated proteins in different groups was evaluated via western blot. (E-F) Transwell and wound healing assays were applied to analyze the migration ability of transfected cells. Scale bar $=100 \mu \mathrm{m}$. (G) The expression of migration-related molecular markers in different groups was analyzed via western blot. The full-length gels for western blot data in Figures S2D and S2G were presented in Supplementary Figure $8 .{ }^{* *} p<0.01$.

Additional file 3: Supplementary Figure 3. (A) qRT-PCR analysis indicated the upregulation of GOLM1 in prostate cancer tissue samples in contrast to peri-tumor samples. (B-E) The immunoblots in Figs. 3c, g, j and $4 \mathrm{~b}$ was quantified. (F) The mRNA and protein levels of GOLM1 in different groups were detected via qRT-PCR and western blot. (G) The expression of proliferation-related proteins in different groups was evaluated via western blot. $(H)$ The quantification of immunoblots in Fig. $4 \mathrm{f}$ was displayed. (I) The expression of migration-related molecular markers in different groups was analyzed via western blot. The full-length gels for western blot data in Figures S3F, S3G and S3I were presented in Supplementary Figure $9 .{ }^{*} p<0.01$.

Additional file 4: Supplementary Figure 4. The full-length gel images of western blots in Fig. 1g.

Additional file 5: Supplementary Figure 5. The full-length gel images of western blot data in Fig. 3c, $g$ and $j$.

Additional file 6: Supplementary Figure 6. The full-length images of western blots in Fig. $4 \mathrm{~b}$ and $\mathrm{f}$.

Additional file 7: Supplementary Figure 7. The full-length images of western blot data in Supplementary Figure $1 \mathrm{C}$ and $\mathrm{E}$.

Additional file 8: Supplementary Figure 8. The full-length images of western blot data in Supplementary Figure 2D and G.

Additional file 9: Supplementary Figure 9. The full-length images of western blot data in Supplementary Figure 3F, G and I.

Additional file 10: Supplementary file 1. The enlarged images of picture data in Fig. 1e, $\mathrm{f}, \mathrm{h}$ and $\mathrm{i}$.

Additional file 11: Supplementary file 2. The enlarged images of picture data in Figs. 2b, 4e, $g$ and $h$.

\section{Abbreviations}

IncRNAs: Long non-coding RNAs; CCK-8: Cell Counting Kit-8; RIP: RNA immunoprecipitation; qRT-PCR: Quantitative real-time PCR;

FISH: Fluorescence in situ hybridization assay; EdU: 5'-ethynyl-2'-deoxyuridine incorporation assay; TUNEL: Transferase-mediated dUTP nick end labeling staining; LINC00992: LncRNA long intergenic non-protein coding RNA 992; GOLM1: Golgi membrane protein 1

\section{Acknowledgements}

We thank all our experimenters for their efforts.

\section{Authors' contributions}

This research project was mainly designed by JH, XD and KB. JA and GZ cooperated to carry out the functional assays and molecular mechanism assays. Besides, the Results and Figure legends sections of the manuscript were accomplished by $H T$, and XZ was in charge of the statistical analyses and the rest part of the manuscript. All authors have read and approved the manuscript. 


\section{Funding}

This work was supported by 2016 Anhui Province Human Society Hall Preferential Subsidy Program of Innovative Project for Overseas Students (2016RSL18), which only provided funding support and did not participate in specific research.

\section{Availability of data and materials}

All data generated or analysed during this study are included in this published article and its supplementary information files.

\section{Ethics approval and consent to participate}

After obtaining the informed consents from all participants, experiments regarding human were conducted under the approval of the Ethics Committee of the First Affiliated Hospital of Kunming Medical University. Animal study was performed in line with the Basel Declaration outlines fundamental principles and the ethical guidelines published by the International Council for Laboratory Animal Science. Also, the animal experiments were approved by the Ethics Committee of the First Affiliated Hospital of Kunming Medical University. However, ethical approval was not required for the use of all cell lines in this study.

\section{Consent for publication}

The approvals from all authors are gained for its submission.

\section{Competing interests}

The authors declare that they have no competing interests.

\section{Author details}

${ }^{1}$ Department of Urology, the First Affiliated Hospital of Kunming Medical University, Kunming 650032, Yunnan, China. ${ }^{2}$ Department of Urology, the First Affiliated Hospital of Anhui University of Chinese Medicine, Hefei 230031, Anhui, China. ${ }^{3}$ Department of Urology, Xiyuan Hospital, China Academy of Chinese Medical Sciences, Beijing 100091, China. ${ }^{4}$ Department of Urology, Akita University School of Medicine, Akita 010-8543, Japan. ${ }^{5}$ Department of Urology, the Second Affiliated Hospital of Bengbu Medical College, 220 Hongye Road, Bengbu 233000, Anhui, China.

Received: 14 November 2019 Accepted: 6 July 2020

Published online: 11 August 2020

\section{References}

1. Siegel RL, Miller KD, Jemal A. Cancer statistics, 2019. CA Cancer J Clin. 2019; 69(1):7-34.

2. Sturge J, Caley MP, Waxman J. Bone metastasis in prostate cancer: emerging therapeutic strategies. Nat Rev Clin Oncol. 2011;8(6):357-68.

3. Smith MR, Cook R, Lee KA, Nelson JB. Disease and host characteristics as predictors of time to first bone metastasis and death in men with progressive castration-resistant nonmetastatic prostate cancer. Cancer. 2011; 117(10):2077-85

4. Guttman M, Amit I, Garber M, French C, Lin MF, Feldser D, et al. Chromatin signature reveals over a thousand highly conserved large non-coding RNAs in mammals. Nature. 2009;458(7235):223-7.

5. Schmitz SU, Grote P, Herrmann BG. Mechanisms of long noncoding RNA function in development and disease. Cell Mol Life Sci. 2016;73(13):2491509.

6. Wang C, Jing J, Cheng L. Emerging roles of non-coding RNAs in the pathogenesis, diagnosis and prognosis of osteosarcoma. Investig New Drugs. 2018;36(6):1116-32.

7. Bhan A, Soleimani M, Mandal SS. Long noncoding RNA and Cancer: a new paradigm. Cancer Res. 2017;77(15):3965-81.

8. Renganathan A, Felley-Bosco E. Long noncoding RNAs in Cancer and therapeutic potential. Adv Exp Med Biol. 2017;1008:199-222.

9. Bai J, Yao B, Wang L, Sun L, Chen T, Liu R, et al. IncRNA A1BG-AS1 suppresses proliferation and invasion of hepatocellular carcinoma cells by targeting miR-216a-5p. J Cell Biochem. 2019;120(6):10310-22.

10. Li Q, Lu J, Xia J, Wen M, Wang C. Long non-coding RNA LOC730100 enhances proliferation and invasion of glioma cells through competitively sponging miR-760 from FOXA1 mRNA. Biochem Biophys Res Commun. 2019;512(3):558-63.

11. Guo Z, Zhang J, Fan L, Liu J, Yu H, Li X, et al. Long noncoding RNA (IncRNA) small Nucleolar RNA host gene 16 (SNHG16) predicts poor prognosis and
Sorafenib resistance in hepatocellular carcinoma. Med Sci Monit. 2019;25: 2079-86.

12. Wang Y, Wang Z, Xu J, Li J, Li S, Zhang M, et al. Systematic identification of non-coding pharmacogenomic landscape in cancer. Nat Commun. 2018; 9(1):3192.

13. Ye Y, Li S-L, Wang S-Y. Construction and analysis of mRNA, miRNA, IncRNA, and TF regulatory networks reveal the key genes associated with prostate cancer. PLoS One. 2018;13(8):e0198055.

14. Tsai MC, Manor O, Wan Y, Mosammaparast N, Wang JK, Lan F, et al. Long noncoding RNA as modular scaffold of histone modification complexes. Science. 2010;329(5992):689-93.

15. Tripathi V, Ellis JD, Shen Z, Song DY, Pan Q, Watt AT, et al. The nuclearretained noncoding RNA MALAT1 regulates alternative splicing by modulating SR splicing factor phosphorylation. Mol Cell. 2010;39(6):925-38.

16. Sen R, Ghosal S, Das S, Balti S, Chakrabarti J. Competing endogenous RNA the key to posttranscriptional regulation. Sci World J. 2014;2014:896206.

17. Tay Y, Rinn J, Pandolfi PP. The multilayered complexity of ceRNA crosstalk and competition. Nature. 2014;505(7483):344-52.

18. Feng Y, Ma J, Fan H, Liu M, Zhu Y, Li Y, et al. TNF-alpha-induced IncRNA LOC105374902 promotes the malignant behavior of cervical cancer cells by acting as a sponge of miR-1285-3p. Biochem Biophys Res Commun. 2019; 513(1):56-63.

19. Cui Z, Luo Z, Lin Z, Shi L, Hong Y, Yan C. Long non-coding RNA TTN-AS1 facilitates tumorigenesis of papillary thyroid cancer through modulating the miR-153-3p/ZNRF2 axis. J Gene Med. 2019;21(5):e3083.

20. Yang J, Liang B, Hou S. TMPO-AS1 promotes cervical cancer progression by upregulating RAB14 via sponging miR-577. J Gene Med. 2019;21(11):e3125.

21. Pu J, Wei H, Tan C, Qin B, Zhang Y, Wang A, et al. Long noncoding RNA SNHG14 facilitates hepatocellular carcinoma progression through regulating miR-4673/SOCS1. Am J Transl Res. 2019;11(9):5897-904.

22. Zhang $M$, Weng $W$, Zhang $Q$, Wu $Y$, Ni S, Tan $C$, et al. The IncRNA NEAT1 activates Wnt/B-catenin signaling and promotes colorectal cancer progression via interacting with DDX5. J Hematol Oncol. 2018;11(1):113.

23. Zheng JF, Guo NH, Zi FM, Cheng J. Long non-coding RNA H19 promotes tumorigenesis of multiple myeloma by activating BRD4 signaling by targeting miR-152-3p. Mol Cell Biol. 2019;40(3):e00382-19.

24. Chen J, Yu Y, Li H, Hu Q, Chen X, He Y, et al. Long non-coding RNA PVT1 promotes tumor progression by regulating the miR-143/HK2 axis in gallbladder cancer. Mol Cancer. 2019;18(1):33.

25. Yan G, Ru Y, Wu K, Yan F, Wang Q, Wang J, et al. GOLM1 promotes prostate cancer progression through activating PI3K-AKT-mTOR signaling. Prostate. 2018;78(3):166-77.

26. Kojima S, Enokida H, Yoshino H, Itesako T, Chiyomaru T, Kinoshita T, et al. The tumor-suppressive microRNA-143/145 cluster inhibits cell migration and invasion by targeting GOLM1 in prostate cancer. J Hum Genet. 2014;59(2): 78-87.

27. Li W, Wang X, Li B, Lu J, Chen G. Diagnostic significance of overexpression of Golgi membrane protein 1 in prostate cancer. Urology. 2012;80(4):952 e1-7.

28. Varambally S, Laxman B, Mehra R, Cao Q, Dhanasekaran SM, Tomlins SA, et al. Golgi protein GOLM1 is a tissue and urine biomarker of prostate cancer. Neoplasia (New York, NY). 2008;10(11):1285-94.

29. Tolkach Y, Merseburger A, Herrmann T, Kuczyk M, Serth J, Imkamp F. Signatures of adverse pathological features, androgen insensitivity and metastatic potential in prostate Cancer. Anticancer Res. 2015;35(10):5443-51.

30. Kong Q, Qiu M. Long noncoding RNA SNHG15 promotes human breast cancer proliferation, migration and invasion by sponging miR-211-3p. Biochem Biophys Res Commun. 2018;495(2):1594-600.

31. Li Y, Luo H, Xiao N, Duan J, Wang Z, Wang S. Long noncoding RNA SChLAP1 accelerates the proliferation and metastasis of prostate Cancer via targeting miR-198 and promoting the MAPK1 pathway. Oncol Res. 2018; 26(1):131-43.

32. Xu W, Chang J, Du X, Hou J. Long non-coding RNA PCAT-1 contributes to tumorigenesis by regulating FSCN1 via miR-145-5p in prostate cancer. Biomed Pharmacother. 2017;95:1112-8.

33. Ding $X$, Deng $G$, Liu J, Liu B, Yuan F, Yang $X$, et al. GOLM1 silencing inhibits the proliferation and motility of human glioblastoma cells via the Wnt/betacatenin signaling pathway. Brain Res. 2019;1717:117-26.

34. Xu R, Ji J, Zhang X, Han M, Zhang C, Xu Y, et al. PDGFA/PDGFRalpharegulated GOLM1 promotes human glioma progression through activation of AKT. J Exp Clin Cancer Res. 2017;36(1):193. 
35. Zhang R, Zhu Z, Shen W, Li X, Dhoomun DK, Tian Y. Golgi membrane protein 1 (GOLM1) promotes growth and metastasis of breast Cancer cells via regulating matrix Metalloproteinase-13 (MMP13). Med Sci Monit. 2019; 25:847-55.

36. Aruna LLM. Overexpression of golgi membrane protein 1 promotes nonsmall-cell carcinoma aggressiveness by regulating the matrix metallopeptidase 13. Am J Cancer Res. 2018;8(3):551-65.

37. Zhang S, Ge W, Zou G, Yu L, Zhu Y, Li Q, et al. MiR-382 targets GOLM1 to inhibit metastasis of hepatocellular carcinoma and its down-regulation predicts a poor survival. Am J Cancer Res. 2018;8(1):120-31.

38. Yang L, Luo P, Song Q, Fei X. DNMT1/miR-200a/GOLM1 signaling pathway regulates lung adenocarcinoma cells proliferation. Biomed Pharmacother. 2018:99:839-47.

39. Liu X, Chen L, Zhang T. Increased GOLM1 expression independently predicts unfavorable overall survival and recurrence-free survival in lung adenocarcinoma. Cancer Control. 2018;25(1):1073274818778001.

\section{Publisher's Note}

Springer Nature remains neutral with regard to jurisdictional claims in published maps and institutional affiliations.

Ready to submit your research? Choose BMC and benefit from:

- fast, convenient online submission

- thorough peer review by experienced researchers in your field

- rapid publication on acceptance

- support for research data, including large and complex data types

- gold Open Access which fosters wider collaboration and increased citations

- maximum visibility for your research: over $100 \mathrm{M}$ website views per year

At $\mathrm{BMC}$, research is always in progress.

Learn more biomedcentral.com/submissions 